EPJ Web of Conferences 108, 02035 (2016)

DOI: $10.1051 /$ epjconf/201610802035

(C) Owned by the authors, published by EDP Sciences, 2016

\title{
An Efficient Method to Study Nondiffusive Motion of Brownian Particles
}

\author{
Vladimír Lisý1,2,a and Jana Tóthová2,b \\ ${ }^{1}$ Laboratory of Radiation Biology, Joint Institute for Nuclear Research, 141980 Dubna, Moscow Region, \\ Russia \\ ${ }^{2}$ Department of Physics, Faculty of Electrical Engineering and Informatics, Technical University of Košice, \\ Park Komenského 2, 04200 Košice, Slovakia
}

\begin{abstract}
The experimental access to short timescales has pointed to the inadequacy of the standard Langevin theory of the Brownian motion (BM) in fluids. The hydrodynamic theory of the BM describes well the observed motion of the particles; however, the published approach should be improved in several points. In particular, it leads to incorrect correlation properties of the thermal noise driving the particles. In our contribution we present an efficient method, which is applicable to linear generalized Langevin equations describing the BM of particles with any kind of memory and apply it to interpret the experiments where nondiffusive BM of particles was observed. It is shown that the applicability of the method is much broader, allowing, among all, to obtain efficient solutions of various problems of anomalous BM.
\end{abstract}

\section{Introduction}

In several recent papers, see the review article [1], the positional fluctuations of Brownian microspheres were studied by confining the particles in an optical trap. These experiments clearly show the inadequacy of the standard Langevin theory [2] to describe the Brownian motion (BM) in fluids. So, in contrast to the commonly assumed overdamped motion, experimental access to short timescales has revealed a resonance peak in the spectrum of the particle fluctuations in a liquid [3], the transition from ballistic to diffusive BM has been observed [4] and even, as reported in [5], the instantaneous velocity of a Brownian particle has been measured for the first time in air. For the history and more references on the experiments and theory of the BM we refer to [1, 6-8]. The obtained results are well described by the hydrodynamic theory of the BM in harmonic potentials [9] and are interesting also because they can be considered as measurements of the "color" of the thermal noise driving the Brownian particles through collisions with the fluid molecules $[3,10,11]$. However, the interpretation based on the work [9] should be improved. In particular, the reported correlation properties of the thermal random force are not correct. In this contribution we present a simple but efficient method of solving the linear generalized Langevin equations aimed at describing the BM with any kind of memory. The solution of this equation leads to properties of the thermal random force which significantly

\footnotetext{
a e-mail: vladimir.lisy@tuke.sk

be-mail: jana.tothova@tuke.sk
} 
differ from those found in the literature $[3,10,11]$. The approach we use comes back to the old work [12] where the hydrodynamic theory of the BM was built for the first time. We give the description of the method, its proof, and illustrate its efficiency on several problems related to the normal and anomalous BM.

\section{A simple method to solve the generalized Langevin equation}

Let us consider the popular generalized Langevin equation,

$$
M \dot{v}+\int_{0}^{t} \Gamma\left(t-t^{\prime}\right) v\left(t^{\prime}\right) \mathrm{d} t^{\prime}=\eta(t),
$$

where, $M$ is the mass of the Brownian particles and $v(t)=\dot{x}(t)$ is its velocity. The kernel $\Gamma(t)$ describes the memory in the system and $\eta(t)$ is a stochastic force with zero mean. The fluctuationdissipation theorem dictates that the kernel $\Gamma$ must be connected to the noise $\eta(t)$ by the relation $\langle\eta(t) \eta(0)\rangle=k_{B} T \Gamma(t)$ (at $t>0$ ). This equation can be immediately solved as follows. In [13], Vladimirsky considered a method for the evaluation of the averaged products of two thermally fluctuating quantities related to different moments of time. The difficult task of the calculation of such means using the Gibbs method was reduced to a much simpler solution of equations of motion. The obtained rules of finding the time correlation functions are thus consistent with the general principles of statistical mechanics and simultaneously with the linear phenomenological equations of motion for the studied quantities. If we are interested in finding the mean square displacement (MSD) of the particle, $X(t)=\left\langle\Delta x^{2}(t)\right\rangle=\left\langle[x(t)-x(0)]^{2}\right\rangle$, according to [13] we have merely to substitute $X(t)$ for $x(t)$ in (1) by and replace the stochastic force driving the particle with the constant "force" $f=2 k_{B} T$, where $k_{B}$ is the Boltzmann constant and $T$ the temperature. Of course, having the dimension of energy, $f$ is not a true force; it only plays this role in the equation for the particle "position" $X(t)$. This fictitious force is considered to begin to act on the particle at the time $t=0$. Up to this moment the particle is at rest so that the equation of motion must be solved with the initial conditions $X(0)=V(0)=0$, where we have introduced the "velocity" $V(t)=\mathrm{d} X(t) / \mathrm{d} t$. Obviously, also the condition $\dot{V}(0)=2 k_{B} T / M$ must hold. It is important that at the initial moment the whole studied system is assumed to be in a state of thermodynamic equilibrium. From the point of view of the macroscopic equations of motion, such an initial state corresponds to the long-lasting rest of the system. Thus the values of all the parameters entering the equations of motion are specified at any $t>0$. This allows one to study not only equations for $x(t)$ in the form of differential equations (that require only the specifications of the initial values of the quantities) but also any Volterra-type integral equations. Such equations can describe systems for which the whole history of their motion is important. The discussed method is not limited to a concrete law of the aftereffect. Its advantage is that it is applicable to any kind of memory in the studied (linear) system and the only requirement is the linearity and existence of a unique solution at the given initial conditions. Equation (1) can be thus rewritten in the form

$$
M \dot{V}+\int_{0}^{t} \Gamma\left(t-t^{\prime}\right) V\left(t^{\prime}\right) \mathrm{d} t^{\prime}=2 k_{B} T,
$$

which, with the mentioned initial conditions, can be easily solved, e.g., using the Laplace transformation $\tilde{V}(s)=\mathcal{L}\{V(t)\}, \tilde{\phi}(s)=\mathcal{L}\{\phi(t)\}$, and the relation $s \tilde{V}(s)=2 \tilde{\phi}(s)$. Here $\phi(t)=\langle v(t) v(0)\rangle$ is the velocity autocorrelation function (VAF). From the equipartition theorem we have for $\phi(t)$ the initial 
condition $\phi(0)=\left\langle v^{2}\right\rangle=k_{B} T / M$. Other time correlation functions are connected to $\phi(t)$ by the relations $\phi(t)=\mathrm{d} D(t) / \mathrm{d} t$, where $D(t)=V(t) / 2$ is the time-dependent diffusion coefficient in the chosen direction and $X(t)=2 \int_{0}^{t}(t-s) \phi(s) \mathrm{d} s$ [14], so that $\phi(t)=\ddot{X}(t) / 2$. Equation (2) can be derived as follows. We multiply the equation (1) by $v(0)$ and average it. This gives

$$
M \dot{\phi}+\int_{0}^{t} \Gamma\left(t-t^{\prime}\right) \phi\left(t^{\prime}\right) \mathrm{d} t^{\prime}=0
$$

The equivalence between (2) and (3) is seen from the above relations between the Laplace transformed quantities $V$ and $\phi$. When external forces are included in (1), the described method of its solution is applicable as well, assuming the equations remain linear. One just has to represent the distance a particle moves in time as an integral of its velocity $v(t), x(t)-x(0)=\int_{0}^{t} v(s) \mathrm{d} s$. Note that in our approach leading to (3) we do not use the initial condition $v(0)=0[9,15,16]$ for the stochastic variable $v(t)$.

Another method of solving the generalized Langevin equation, based on the linear response theory [17] and leading to the correct result (see however the discussion in [8]), was presented in [15]. The velocity of the Brownian particle was expressed as $v(t)=\left(k_{B} T\right)^{-1} \int_{0}^{t} \phi\left(t-t^{\prime}\right) \eta\left(t^{\prime}\right) \mathrm{d} t^{\prime}$, with the Laplace transform $\tilde{v}(s)=\left(k_{B} T\right)^{-1} \tilde{\phi}(s) \tilde{\eta}(s)$. Since the Laplace transform of Eq. (1) is $M s \tilde{v}(s)-M v(0)+$ $\tilde{\Gamma}(s) \tilde{v}(s)=\tilde{\eta}(s)$, we obtain

$$
\mathcal{L}\{v(t)\}=\tilde{\phi}(s)=\frac{k_{B} T}{M s+\tilde{\Gamma}(s)}=\frac{s \tilde{V}(s)}{2}=\frac{s^{2} \tilde{X}(s)}{2},
$$

in agreement with the solution of (3). The solution of the standard Langevin equation $M \dot{v}+\gamma v=\eta(t)$ (now with the white noise $\eta(t)$ and $\gamma$ being a constant friction coefficient) is the same if $\tilde{\Gamma}$ is replaced by $\gamma$. The inverse transformation gives $\dot{\phi}(t)=-\tau^{-1} \phi(t)$ with the solution $\phi(t)=\left(k_{B} T / m\right) \exp (-t / \tau)$, $\tau=M / \gamma$ being the relaxation time due to viscous friction. The solution of (4) requires knowledge of the system memory. However, independently of a concrete form of $\Gamma(t)$, at short times the generalized Langevin equation describes the ballistic motion, $X(t) \approx k_{B} T t^{2} / M$ as $t \rightarrow 0[7,18]$. The longtime properties of the MSD can be related to the behavior of the Laplace transform of the kernel $\Gamma$, $\tilde{\Gamma}=\mathcal{L}\{\Gamma(t)\}$ at small $s[19]$. From (4) it follows that if at small $s$ the kernel $\tilde{\Gamma}$ behaves as $\tilde{\Gamma} \propto s^{v}$ and the root MSD at long times is $X(t) \propto t^{\alpha}$, then there are two possibilities: i) if $v<1$, then $\alpha=v+1$, and the velocity autocorrelation function $\phi(t)$ decays to zero as $t \rightarrow \infty$ (this corresponds to the irreversibility condition and, consequently, the ergodicity of the variable $v$ [20]), ii) if $v \geq 1$, then $\alpha=2$ (ballistic motion) and $\phi(t)$ converges to a constant as $t \rightarrow \infty$. We have sub-diffusion $(\alpha<1)$ if $v<0$ and super-diffusion $(\alpha>1)$ if $v>0$. The Einstein diffusion corresponds to $v=0$.

As an example, let us specify the memory kernel by choosing it in the Ornstein-Uhlenbeck form $\Gamma(t)=\left(\gamma^{2} / m\right) \exp (-\gamma t / m)$. Then the force $\eta(t)=m \dot{u}(t)$ corresponds to the solution of the usual Langevin equation $m \dot{u}(t)+\gamma u(t)=f(t)$. Here $u(t)$ is the velocity of a Brownian particle and $f(t)$ the stochastic (white noise) force. The motion of the particles with mass $M$ can be thus interpreted as being induced by the force $\eta$ caused by the particles of mass $m$. Using (4) or directly solving (2) by the Laplace transformation, one can straightforwardly obtain the solution for any of the relevant correlation functions. For example, the time-dependent diffusion coefficent is

$$
D(t)=\frac{k_{B} T}{\gamma}\left\{1-\frac{1}{4 \mu} \exp (-\gamma t / 2 m)\left[(1+\mu)^{2} \exp (\gamma \mu t / 2 m)-(1-\mu)^{2} \exp (-\gamma \mu t / 2 m)\right]\right\}
$$


with $\mu=\sqrt{1-4 m / M}$. If $M<4 m$, this solution describes damped oscillations. When $\gamma / m \rightarrow 0$ ( $\Gamma=\gamma^{2} / m=$ const $)$, we come to a special case [21]:

$$
D(t) \approx \frac{k_{B} T}{\sqrt{\Gamma M}} \sin \left(\sqrt{\frac{\Gamma}{M}} t\right) .
$$

\section{Hydrodynamic Langevin equation for the Brownian motion}

It is long known [12] that the standard Langevin equation describing the BM of particles is valid only under limited conditions. In a more general theory, instead of the Stokes friction the resistance force must reflect the memory in the BM. Assuming incompressible fluids, this is the Boussinesq-Basset force [22]. The equation for the velocity $v(t)=\mathrm{d} x / \mathrm{d} t$ of Brownian particles then has the form

$$
M^{*} \dot{v}(t)+\gamma v(t)+\int_{t_{0}}^{t} \Gamma\left(t-t^{\prime}\right) \dot{v}\left(t^{\prime}\right) \mathrm{d} t^{\prime}=\xi(t),
$$

where $\Gamma(t)=\gamma\left(\tau_{R} / \pi t\right)^{1 / 2}$ with $\tau_{R}=R^{2} \rho / \eta, R$ is the radius of the particle, $\rho$ is the fluid density and $\eta$ its viscosity, $\gamma=6 \pi \eta R$ is the Stokes friction coefficient, and $M^{*}=M+M_{s} / 2$, with $M_{s}$ being the mass of the fluid displaced by the particle of mass $M$. The particle is in thermal equilibrium with the liquid. The initial time moment is infinitely remote from $t, t_{0}=-\infty$. When the particle is in an external harmonic field, the force $-K x(t)$, where $x(t)$ is the particle displacement from the trap centre, should be added in the right hand side of (7). In the traditional LE the thermal noise force $\xi(t)$ is white. This is not the case here since, due to the fluctuation-dissipation theorem [17] at different times, the values of $\xi(t)$ correlate. The solution of (7), e.g., for the VAF, is [12, 23],

$$
\phi(t)=\frac{k_{B} T}{M^{*}} \frac{1}{\lambda_{1}-\lambda_{2}}\left[\lambda_{1} \exp \left(\lambda_{1}^{2} t\right) \operatorname{erfc}\left(-\lambda_{1} \sqrt{t}\right)-\lambda_{2} \exp \left(\lambda_{2}^{2} t\right) \operatorname{erfc}\left(-\lambda_{2} \sqrt{t}\right)\right] .
$$

At short times $X(t \rightarrow 0) \sim k_{B} T t^{2} / M^{*}$, and as $t \rightarrow \infty$ the motion is diffusive, $X(t)=2 D t$, where $D=k_{B} T / \gamma$ is the Einstein diffusion coefficient of the particle. The result (8) has been obtained also in $[3,9,15,16,24]$. In these works, the lower integration limit in (7) is $t_{0}=0$ and the zero initial condition for the particle velocity was assumed. In [16], both the projection of the particle position vector $x(t)$ on the $x$ axis, and the velocity $v(t)$ were assumed zero at $t=0: x(0)=v(0)=0$. It was argued that, within the approximation of small thermal displacements, there is no loss of generality in choosing these trivial conditions. Similarly, in [9] one finds that the velocity of the particle is determined by its velocity at earlier times via backflow effects in the fluid, but it is assumed that the particle is at the equilibrium position $x(0)=0$ and at rest for $t \leq 0$. The latter condition is used also in [15], where the same solution as in [16] for the VAF and MSD has been independently obtained within the linear response theory [17]. In our opinion, the simultaneous use of the conditions $x(0)=0$ and $v(0)=0$ is not consistent with the randomness of the variables $x(t)$ and $v(t)$. Moreover, it is either assumed, or it follows from the calculations in all the cited works, that the equipartition theorem holds, i.e. that $\left\langle v^{2}(0)\right\rangle$ is nonzero. It can be shown that if the above improper assumptions are abandoned, the correct (normal) diffusion can be obtained only if the random force at $t>0$ is correlated with the particle velocity at $t=0$. That is, if $t_{0}=0$ and the random force in (7) is $\zeta(t)$, one must require that $Z(t)=\langle\zeta(t) v(0)\rangle$ is nonzero at $t>0$ (as distinct from the correlator $\langle\xi(t) v(0)\rangle$, which is zero due to the causality). Acting in the same way as in the preceding section for the generalized Langevin equation, we find that the normal diffusion is obtained if $\tilde{Z}(s) / k_{B} T=-\left(\tau_{R} / \tau^{2} s\right)^{1 / 2}$. In fact, $\zeta(t)$ and $\xi(t)$ from (7) 
are connected by the relation $\zeta(t)=\xi(t)-\int_{-\infty}^{0} \Gamma\left(-t^{\prime}\right) \dot{v}\left(t^{\prime}\right) \mathrm{d} t^{\prime}$. In the same way as in the preceding section one finds the equation for the VAF,

$$
M^{*} \dot{\phi}+\gamma \phi+\int_{0}^{t} \Gamma\left(t-t^{\prime}\right) \dot{\phi}\left(t^{\prime}\right) \mathrm{d} t^{\prime}=Z(t) .
$$

Its solution in the Laplace transform is

$$
\tilde{\phi}(s)=\frac{k_{B} T}{M^{*}} \frac{1+\left(\tau_{R} / s\right)^{1 / 2} \tau^{-1}+\tilde{Z}(s) / k_{B} T}{s+\left(\tau_{R} s\right)^{1 / 2} \tau^{-1}+\tau^{-1}} .
$$

Let us require that in the long time limit the particle is in the diffusion regime with Einstein's MSD proportional to $t$. This may happen only if $\tilde{\phi}(s)$ at $s \rightarrow 0$ tends to a constant, namely, $\tilde{\phi}(s) \rightarrow$ $k_{B} T / \gamma=k_{B} T \tau / M^{*}$. Consequently, $\tilde{Z}(s) / k_{B} T \approx-\left(\tau_{R} / \tau^{2} s\right)^{1 / 2}$ for small $s$. Other terms on the right that could go to zero if $s \rightarrow 0$ must be excluded since, at $s \rightarrow \infty$, the right $t=0$ limit holds provided $s \tilde{\phi}(s) \approx k_{B} T / M^{*}$. The relation $\tilde{Z}(s) / k_{B} T=-\left(\tau_{R} / \tau^{2} s\right)^{1 / 2}$ is thus valid at every $s$. Then the VAF is exactly the Laplace transform of the correct Eq. (8). If it is only required, more generally, that $\phi(t) \rightarrow 0$ as $t \rightarrow \infty$, then one finds that at $s \rightarrow 0$ the correlator $\tilde{Z}(s)$ must behave as $\tilde{Z}(s) \approx A s^{-\mu}$, $\mu<1$, i.e., $Z(t) \approx A t^{-1} / \Gamma(\mu)$, where $0<\mu<1$ and $\Gamma(\mu)$ is the Gamma function. The Einstein diffusion corresponds to $\mu=1 / 2$.

Finally, we note that the discussed methods of solving the generalized Langevin equation (1) presented in $[9,15,16]$ and some other papers lead to the correct results for the correlation functions of the dynamical variables $x$ and $v$, as well as for the "color" of the thermal noise, i.e., the correlation function $\langle\eta(t) \eta(0)\rangle$. However, it does not apply to the hydrodynamic Langevin equation (7). In particular, for (7), these methods give an incorrect time dependence of the function $\langle\xi(t) \xi(0)\rangle$. As argued in $[8,25],\langle\xi(t) \xi(0)\rangle$ in incompressible fluids at long times approaches zero as $\sim t^{-1 / 2}$, instead of $t^{-3 / 2}$ found in $[3,10,11]$.

\section{Conclusions}

The method described in this contribution provides an efficient way to solve the generalized Langevin equation describing the Brownian motion of particles. This stochastic equation, containing usually the convolution of the particle velocity or acceleration with a memory kernel, is converted in one step to an equation for the mean square displacement of the particle or related functions such as the velocity autocorrelation function or the time dependent diffusion coefficient. The obtained equations are then solved by standard methods. It is important that our approach is not limited to the generalized Langevin equations but it allows also solving different linear phenomenological stochastic equations in the frame of the Gibbs statistical physics. Possible applications that are currently of great interest concern not only the discussed motion of the Brownian particles dragged by optical tweezers but also, for example, the anomalous motion of colloidal particles under the influence of various external fields [26], the behavior of mesoscale electric circuits in contact with a thermal bath [27], the motion of magnetic domain walls [28], or the dynamics of polymers [6].

\section{Acknowledgements}

This work was supported by the Agency for the Structural Funds of the EU within the projects NFP 26220120021, 26220120033, and by the grant VEGA 1/0348/15. 


\section{References}

[1] T. Li., M. G. Raizen, Ann. Phys. (Berlin) 525, 281 (2013)

[2] P. Langevin, P. C. R. Acad. Sci. (Paris) 146, 530 (1908)

[3] Th. Franosch et al., Nature 478, 85 (2011)

[4] R. Huang et al., Nature Phys. 7, 576 (2011)

[5] T. Li et al., Science 328, 1673 (2010)

[6] J. Tothova, V. Lisy, Acta Phys. Slovaca 65, 1 (2015)

[7] J. Tothova et al., Eur. J. Phys. 32, 645 (2011)

[8] J. Tothova, L. Glod, V. Lisy, Int. J. Thermophys. 34, 629 (2013)

[9] H. J. H. Clercx, P. P. J. M. Schram, Phys. Rev. A 46, 1942 (1992)

[10] K. Berg-Sørensen, H. Flyvbjerg, New J. Phys. 7, 2 (2005)

[11] A. Jannasch, A. Mahamdeh, E. Schäffer, Phys. Rev. Lett. 107, 228301 (2011)

[12] V. Vladimirsky, Ya. Terletzky, Zh. Eksp. Teor. Fiz. 15, 258 (1945)

[13] V. Vladimirsky, Zh. Eksp. Teor. Fiz. 12, 199 (1942)

[14] P. P. J. M. Schram, I. P. Yakimenko, Physica A 260, 73 (1998)

[15] Karmeshu, J. Phys. Soc. Japan 34, 1467 (1973)

[16] E. J. Hinch, Fluid Mech. 72, 499 (1975)

[17] R. Kubo, Rep. Prog. Phys. 29, 255 (1966)

[18] R. Morgado et al., Phys. Rev. Lett. 89, 100601 (2002)

[19] M. Abramowitz, I. A. Stegun, Handbook of Mathematical Functions (National Bureau of Standards, Washington, DC, 1964)

[20] L. C. Lapas et al., Phys. Rev. Lett. 101, 230602 (2008)

[21] I. Goychuk, Phys. Rev. E 80, 046125 (2009)

[22] J. Boussinesq, P. C. R. Acad. Sci. (Paris) 100, 935 (1885)

[23] V. Lisy, J. Tothova, arXiv:cond-mat/0410222 [cond-mat.stat-mech]

[24] F. Mainardi, P. Pironi, Extracta Mathematicae 10, 140 (1996)

[25] J. Tothova, L. Glod, V. Lisy, arXiv:1304.4183 [cond-mat.stat-mech]

[26] H. Löwen, J. Phys.: Cond. Mat. 21, 474203 (2009)

[27] N. Garnier, S. Ciliberto, Phys. Rev. E 71, 060101(R) (2005)

[28] E. Saitoh et al., Nature 432, 203 (2004) 